\begin{tabular}{|l|l|l|l|l|}
\hline Cuadernos de Investigación Geográfica & 2000 & No 26 & pp. 113-130 & ISSN 0211-6820 \\
\hline
\end{tabular}

(c) Universidad de La Rioja

\title{
HIDROLOGÍA SUPERFICIAL EN AMBIENTES ADEHESADOS, CUENCA EXPERIMENTAL GUADALPERALÓN
}

\author{
S. SCHNABEL \\ B. MATEOS RODRÍGUEZ \\ Departamento de Geografía, Universidad de Extremadura, Avda. de la Universidad, 10071 Cáceres \\ C. electrónico: sschnab@geot.unex.es
}

RESUMEN: Se presentan los principales resultados de los estudios bidrológicos desarrollados en la cuenca experimental Guadalperalón, ejemplo de las debesas en las penillanuras del suroeste español. Se abarcaron temas como la producción de escorrentía a diferentes escalas espaciales, la interceptación de lluvia por las encinas y el balance de agua. Se analiza la generación de escorrentía a escala de vertiente. Ésta se encuentra relacionada con las unidades edafo-vegetales existentes. Coeficientes de escorrentía bajos se observan debajo de las copas de los árboles, en zonas de pastizal mejorado (majadeo), $y$ en los pies de vertientes $y$ fondos de vaguada, donde existe un relleno de sedimento fluvio-coluvial. Los suelos poco profundos y degradados muestran capacidad de infiltración escasa. Además, durante sequías prolongadas, cuando la cubierta vegetal es reducida debido al pastoreo continuo, la superficie del suelo tiene un comportamiento bidrofóbico. La bumedad antecedente es un factor importante. Grandes cantidades de escorrentía se generan cuando el evento es precedido por llu vias cuantiosas y suelos saturados. En esta situación todas las unidades espaciales demuestran coeficientes de escorrentía altos. Consecuentemente la humedad antecedente del suelo es decisivo en la producción de caudal. Durante años con lluvias por debajo de la media domina el flujo hortoniano debido a la discontinuidad de la escorrentía por la reinfiltración de agua en las vaguadas rellenas de sedimento. Por el contrario, durante años con precipitaciones abundantes, estas áreas se saturan y las aportaciones son elevadas. Como resultado los coeficientes de escorrentía son altamente variables, con valores muy bajos durante años secos (1.5\%) y valores moderados durante años búmedos (15.9\%).

ABSTRACT: Research was carried out in the Guadalperalón catchment in Extremadura, Spain, between 1990 and 1997 in order to understand the bydrological and erosional processes operating in semi-arid open woodlands with silvo-pastoral landuse (debesas). The present paper presents a summary of the major results of the project, including investigations of runoff production at different spatial scales, rainfall interception of bolm oaks, and the water balance of the catchment. At the billslope scale variations of runoff generation could be related to existing soil-vegetation units, which are typical for the peneplain landscape of $S W$ Iberia. Low runoff coefficients are observed below tree canopies, in areas with improved pastures, as well as at footslopes and small valley bottoms with fluvio-colluvial sediment fill. Antecedent soll moisture conditions are 
shown to be of major importance. The shallow and degraded solls bave low infiltration capacity, and, additionally, during prolonged droughts, when ground cover is strongly reduced due to continuous grazing, water repellency was observed. Large amounts of overland flow are produced during events preceded by bigh rainfall amounts and saturated soil conditions. In this situation all spatial units show bigh runoff coefficients. As a consequence antecedent soil moisture conditions are decisive for runoff production. During years with below average rainfall Hortonian overland flow dominates and water flow is discontinuous with reinfiltration in the sediment fill of footslopes and valley bottoms. During years with above average rainfall these areas become saturated, and large amounts of runoff are produced (saturated overland flow). As a result runoff coefficients vary strongly, with very low values during dry years (1.5\%) and moderate values during bumid years (15.9\%).

Palabras clave: Escorrentía superficial, Caudal, Interceptación, Balance de agua, Dehesa.

Key words: Overland flow, Discharge, Interception, Water balance, Silvo-pastoral systems, Semi-arid areas.

\section{Introducción}

Durante el período comprendido entre los años 1990 y 1998 se desarrollaron estudios sobre los procesos hidrológicos y erosivos en la cuenca experimental Guadalperalón, ejemplo de una explotación de dehesa, enmarcada en relieve de penillanura. El término dehesa y su sinónimo portugués 'montado' es aplicado aquí para los sistemas agrosilvopastoriles, de uso múltiple, donde destaca la existencia de un arbolado disperso. Es un ecosistema semi-natural en el que dominan árboles esclerófitos de hoja perenne, principalmente Quercus rotundifolia y Quercus suber. Dicho término implica siempre la presencia de animales domésticos, cuya alimentación se basa principalmente en la vegetación herbácea, aunque también incluye los arbustos y el ramón de los árboles (Montoya Oliver et al., 1988). El aprovechamiento ganadero es desde el punto de vista económico el más importante en la actualidad.

El ganado influye de manera directa e indirecta sobre el ecosistema; reduce la proliferación de especies arbustivas, asegurando la dominancia del pastizal; interviene en la composición florística de los pastos y en su biomasa (Holecheck et al., 1989). El pisoteo produce generalmente un aumento de la densidad aparente y una disminución de la porosidad del suelo, reduciendo significativamente la capacidad de retención hídrica y la tasa de infiltración (Gamougoun et al., 1984; Mulholland \& Fullen, 1991). La reducción de la biomasa y de la cobertura del suelo debido al pastoreo causan una disminución de la interceptación de las precipitaciones, un aumento del porcentaje de suelo desnudo, y como consecuencia, menor protección contra el impacto directo de las gotas de lluvia sobre la superficie del suelo. De manera indirecta, la reducción de la biomasa, con la consiguiente reducción del contenido de materia orgánica del suelo, provoca una degradación física del mismo, afectando negativamente la capacidad de infiltración y retención hídrica, y como consecuencia aumentando la cantidad de escorrentía superficial (Imeson, 1988). 
La investigación se justifica por la gran extensión que ocupan las dehesas y montados en la Península Ibérica, con aproximadamente 3 millones de héctareas, así como por su valor económico. En Extremadura el $50 \%$ de la producción agraria corresponde al aprovechamiento de estos espacios. Además, las dehesas tienen una gran diversidad de vida animal y vegetal (Diáz, et al., 1997) y un alto valor cultural y paisajístico. No obstante, existen muy pocos estudios sobre temas relacionados con la degradación del suelo y los procesos hidrológicos y geomorfológicos de estos ecosistemas, que han sido castigados por deforestación, podas excesivas, falta de regeneración del arbolado y cargas ganaderas elevadas (Elena, M. et al., 1987; Diáz, et al., 1997).

El presente trabajo describe de manera resumida los estudios hidrológicos llevados a cabo en Guadalperalón, que se han centrado en los siguientes objetivos:

1. Determinación de las unidades espaciales de la cuenca atentiendo a las características morfológicas, edáficas y de vegetación.

2. Relación entre escorrentía superficial, factores edáficos y vegetación.

3. Generación de escorrentía a diferentes escalas espaciales (pedón, vertiente y cuenca).

4. Los efectos de la variabilidad temporal de la precipitación.

5. Papel del árbolado en los procesos hídricos.

6. Balance de agua de la cuenca.

Los resultados relacionados con los fenómenos de erosión laminar en las vertientes y el acarcavamiento en los fondos de valle han sido recogidos con anterioridad en Gómez Amelia \& Schnabel (1992, 1996), Schnabel (1997) y Schnabel et al. (1999).

\section{El área de estudio}

Guadalperalón, con una extensión de 35.4 hectáreas, está situado 22 kilómetros al noreste de la ciudad de Cáceres (39 $33^{\prime} 46^{\prime \prime} \mathrm{N}, 6^{\circ} 7^{\prime} 40^{\prime \prime}$ W). El sustrato de la zona está formado por series pizarroso-grauváquicas, de formación probablemente precámbrica (I.G.M.E., 1987). La cuenca, con una altitud máxima de 403 metros, pertenece a la red del río Almonte, afluente del Tajo. Se halla integrada en la unidad geomorfológica más extensa de Extremadura: la superficie de erosión finimiocena, que se caracteriza por su topografía levemente ondulada (Gómez Amelia, 1985). La red fluvial del Tajo sufre un encajamiento vigoroso que va transmitiéndose a los afluentes, dando a los interfluvios perfiles planos con bordes abruptos. Sin embargo las cabeceras que aparecen en las divisorias, como es el caso de Guadalperalón, ofrecen pendientes escasas y vaguadas rellenas de sedimento que se pierden aguas abajo a medida que la erosión fluvial remontante cobra protagonismo. Las vertientes son en general cortas, con pendientes medias que oscilan entre $10 \%$ en la parte superior y el $21 \%$ hacia la salida de la cuenca. La cuenca presenta forma alargada con una longitud de $1100 \mathrm{~m}$ y un desnivel absoluto de $60 \mathrm{~m}$ (Fig. 1). El cauce principal es de orden 2 . 


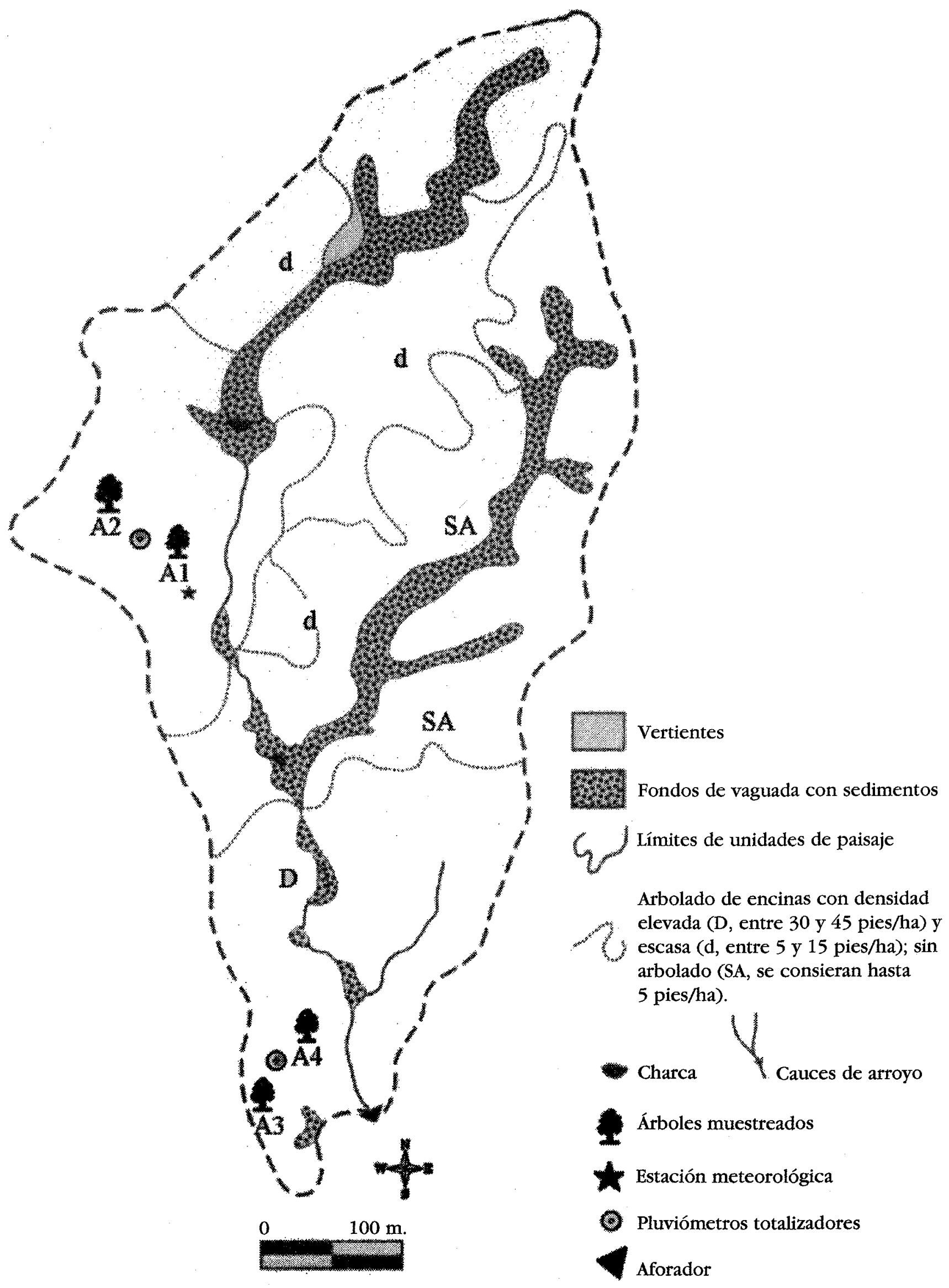

Figura 1. Mapa de la cuenca de Guadalperalón, con densidades de arbolado, localización del aforador, estación meteorológica y los árboles usados en el estudio de la interceptación. Además se indican los fondos de vaguada con sedimentos aluviales. 
Los suelos, de textura franco-limosa y franco-arenosa, son poco profundos, con un espesor que no supera los $30 \mathrm{~cm}$ en la mayor parte de la cuenca. El contenido de materia orgánica es generalmente bajo. Solamente los primeros 2 a $5 \mathrm{~cm}$ poseen cantidades en torno al 3\%, mientras los niveles inferiores no superan el 1\%. En áreas coluviales y fondos de valle los suelos se desarrollan sobre sedimentos cuyo espesor alcanza en algunos puntos $1.5 \mathrm{~m}$. En conjunto, los suelos son poco evolucionados (A-C), y clasificados como Leptosols y Regosols (FAO, 1990).

El clima es mediterráneo, con influencias atlántica y continental (Font Tullot, 1983). Las temperaturas medias oscilan entre los $7.9^{\circ} \mathrm{C}$ en enero y los $25.8^{\circ} \mathrm{C}$ en julio. Análisis de los datos de precipitación del Observatorio de la ciudad de Cáceres han sido presentados por Schnabel (1997 y 1998), cuya serie, disponible desde el año hidrológico de 1907-08, se ha utilizado para caracterizar la pluviometría de la cuenca experimental. Como promedio se registran $516.2 \mathrm{~mm}$ de precipitación, que caen en su mayor parte entre octubre y mayo. La estación seca se extiende entre junio y septiembre. Noviembre y diciembre son los meses que reciben las mayores cantidades, con valores en torno a $70 \mathrm{~mm}$, mientras en julio y agosto las lluvias son casi nulas. La variabilidad de la precipitación anual es alta, con un coeficiente de variación del $30.7 \%$, y un mayor número de años con cantidades inferiores a la media. La variación de las precipitaciones mensuales supera con creces a la anual, destacando el mes de diciembre, que presentan una desviación estándar de $62.2 \mathrm{~mm}$.

La vegetación potencial pertenece a la serie mesomediterránea luso-extremadurense silicícola de la encina (Ladero Álvarez, 1987). En Guadalperalón hay zonas arboladas de $Q$. rotundifolia con una densidad variable de 15 a 40 pies por hectárea y zonas sin arbolado (Fig. 1). El pastizal está formado por especies efímeras de marcado carácter estacional que tienen su máximo crecimiento en primavera y otoño. Se agosta habitualmente a mediados de mayo debido al déficit hídrico del momento. En su composición, con características de pastizal ácido y nitrófilo, dominan las especies anuales.

En el pasado el aprovechamiento de la finca ha sido agrosilvopastoril, con cultivo de cereales en algunas zonas. Desde la década de los 60 la propiedad se dedicó íntegramente al ganado. En algunos períodos se ha simultaneado la cría de cabras y ovejas, junto con cerdos. La finca experimentó una reducción de la carga ganadera a partir de la segunda mitad de los años sesenta. Entre 1989 y 1993 pastaron solamente ovejas (1.1 cabezas/ha). Con el cambio de arrendatarios se introdujeron también vacas, y a partir del otoño de 1995 Guadalperalón experimenta un aumento de la carga ganadera, que se sitúa en 2.6 ovejas y 0.3 vacas/ha. Como actividades secundarias se marcan la caza menor y el carboneo a expensas de la poda.

\section{Metodología}

Las unidades espaciales de la cuenca se determinaron considerando las características morfológicas, edáficas y de vegetación. Ello incluyó la distribución de especies vegetales (Bernet, 1995) y el grado de cobertura. Este último se estudió de manera exhaustiva a lo largo de perfiles de la vertiente situada encima de las tram- 
pas de sedimento de las parcelas de erosión, utilizando un bastidor de $1 \mathrm{~m}^{2}$ y 100 puntos de contacto. Se llevaron a cabo varias series de mediciones para estudiar los cambios temporales de la cobertura (Schnabel, 1997). Durante el año 1994 se efectuaron mediciones mensuales de cobertura, solamente en los dos primeros metros vertiente arriba de cada colector. La profundidad del suelo se ha estimado mediante el levantamiento de una serie de perfiles transversales y longitudinales de la cuenca utilizando una barrena. Para entender el papel de la humedad del suelo en la generación de escorrentía superficial, Ceballos (1999) llevó a cabo una serie de mediciones periódicas de la humedad edáfica superficial $(0-5 \mathrm{~cm})$ aplicando el método gravimétrico. Con la misma periodicidad se determinaron la densidad aparente y la porosidad del suelo.

La entrada de agua se determinó mediante un pluviómetro automático con una resolución de $0.2 \mathrm{~mm}$ (modelo ARG100). Para estudiar la variabilidad espacial de la lluvia se distribuyeron 5 pluviómetros totalizadores en la cuenca, efectuando las lecturas después de cada suceso de lluvia durante el período comprendido entre diciembre de 1994 y agosto de 1996 (Ceballos, 1999).

La capacidad de infiltración de los suelos, así como la escorrentía generada a escala de pedón $\left(0.25 \mathrm{~m}^{2}\right)$ se determinó mediante experimentos con lluvia simulada a una intensidad elevada de $53.6 \mathrm{~mm} \mathrm{~h}^{-1}$. Se realizaron dos series de 20 experimentos con condiciones antecedentes muy contrastadas: en mayo de 1995 con máxima sequedad edáfica (humedad media de $2.7 \%$ en $\operatorname{los} 3 \mathrm{~cm}$ superficiales del suelo) y en febrero de 1996 con suelos muy húmedos (media de $37.5 \%$ ) (Cerdà $e t$ al., 1997; Ceballos, 1999).

Para determinar las pérdidas de suelo y la escorrentía superficial en las vertientes se instalaron 27 parcelas abiertas distribuidas en las diferentes unidades espaciales de la cuenca. Cada parcela consta de una trampa de sedimentos tipo Gerlach de 0.5 $\mathrm{m}$, conectado a un colector. El muestreo se llevó a cabo después de cada evento de precipitación. Existe una serie completa de datos para el período de septiembre 1990 hasta noviembre de 1993 (Schnabel, 1997). Con posterioridad se redujo el número de parcelas a 17, de las cuales se dispone de datos para los años 1994 a 1996. Una importante limitación de la técnica empleada ha sido el desbordamiento de los colectores de escorrentía durante lluvias cuantiosas. El error producido debido a este hecho fue mayor durante años muy húmedos, cuando una parcela se desbordó, como promedio, 6 veces por año ( 1.5 veces durante un año seco).

Los registros automáticos de caudal y de otras variables meteorológicas se efectuaron en intervalos de 5 minutos con dataloggers, modelo Macrologger 7000 de Unidata. La estación meteorológica consta de un pluviómetro de tipo balancín, sensores de temperatura ambiente, humedad relativa del aire, radiación global, y dirección y velocidad del viento.

La descarga acuosa se determinó en la salida de la cuenca mediante un aforador tipo H-flume (USDA, 1979) de tres pies de altura y con una capacidad máxima de $8601 \mathrm{~s}^{-1}$. Un sensor de profundidad de agua (modelo capacitivo de Unidata) permite la determinación del caudal. El H-flume, aunque utilizado con frecuencia en pequeñas cuencas en España, ha resultado no ser el modelo más adecuado en Gua- 
dalperalón, debido a problemas de sedimentación de gravas y arenas. Durante los siete años de funcionamiento, se produjeron en tres ocasiones picos de caudal que superaron la capacidad máxima del aforador.

Se ha estimado la escorrentía superficial generada en una subcuenca de orden 1 mediante la determinación de los cambios del volumen de una charca, situada en la parte alta de la cuenca (Fig. 1), durante los años 1994 y 1995 (Fig. 1). Para ello se estableció una relación entre el nivel de agua y el volumen del vaso de la charca (Ceballos, 1999). Este método es de muy limitada utilidad debido a que no ofrece datos una vez que se llene la charca.

Para determinar el papel del arbolado en el balance hídrico en las dehesas se llevaron a cabo mediciones de la precipitación incidente, trascolación y escorrentía cortical durante 3 años hidrológicos completos (1995-1997) (Mateos \& Schnabel, 1998). Se eligieron 4 encinas, dos de ellas podadas hace más de 25 años y otras dos podadas hace ocho años, con el fin de determinar la influencia de esa práctica sobre la interceptación. Para cuantificar la trascolación se dispusieron 163 pluviómetros simples debajo de las copas de los 4 árboles, distribuidos en una malla regular y con una distancia entre ellos de $1.5 \mathrm{~m}$. El muestreo se efectuó después de cada suceso de precipitación durante los años hidrológicos 1995, 1996, y 1997. Para la medición de la escorrentía cortical se instaló un canal de goma, colocado en espiral alrededor del tronco de cada encina, y que vierte a un colector. La precipitación incidente se determinó con el pluviómetro automático de la estación meteorológica de la cuenca y con dos totalizadores localizados entre los árboles muestreados.

\section{Resultados}

\subsection{Variación temporal de la precipitación}

La precipitación durante el tiempo de estudio ha sido muy variable, con un período prolongado seco durante los primeros años y otro muy húmedo durante los tres últimos años (Tabla 1, Fig. 2). Sequías como la experimentada durante la primera fase de la investigación son un fénomeno común en la zona, que ocurren como promedio cada 8 años (Schnabel, 1997). Las variaciones estacionales e interanuales de la precipitación tuvieron un notable efecto sobre el desarrollo de la vegetación en la cuenca, especialmente sobre el pastizal. Así, el porcentaje de cobertura del suelo sufrió cambios muy marcados, con valores de suelo desnudo que variaron entre un 0\% y un 90\% en algunas zonas de la cuenca (Schnabel, 1997).

\subsection{Variación espacial de la precipitación}

Las diferencias espaciales de las precipitaciones en la cuenca son pequeñas. Los coeficientes de variación anuales son muy bajos (2.3\%) y muestran, además, una tendencia similar durante los dos años estudiados (Ceballos, 1999). Los valores más bajos corresponden a dos pluviómetros situados en zonas altas de la cuenca, donde el viento es más frecuente e intenso; el resto de los pluviómetros se encuentra en posición menos expuesta a los efectos del viento (tramos inferiores de las vertien- 
tes y fondos de valle). Estos resultados coinciden con observaciones de otros autores para pequeñas cuencas en ambientes semiáridos (Sharon, 1970). Se puede afirmar que la variabilidad espacial de las precipitaciones apenas repercute en el balance anual de la cuenca. No obstante, algunos sucesos de lluvia pueden mostrar una variación espacial más elevada debido a su carácter tormentoso, con mayor incidencia sobre la respuesta hidrológica.

Tabla 1: Precipitación mensual y anual en la cuenca Guadalperalón entre 1990 y 1997, y caracterización del año.

\begin{tabular}{lrrrrrrrrr}
\hline & $1990-91$ & $1991-92$ & $1992-93$ & $1993-94$ & $1994-95$ & $1995-96$ & $1996-97$ & $1997-98$ & Media \\
\hline Sep & 24.1 & 16.0 & 27.2 & 21.2 & 16.9 & 11.4 & 65.1 & 86.6 & 33.6 \\
Oct & 95.6 & 45.2 & 95.8 & 125.0 & 64.9 & 10.2 & 51.8 & 66.0 & 69.3 \\
Nov & 93.3 & 11.2 & 12.0 & 70.8 & 67.1 & 96.1 & 80.2 & 355.4 & 98.3 \\
Dic & 20.3 & 65.4 & 46.6 & 4.1 & 17.6 & 151.9 & 166.3 & 126.0 & 74.8 \\
Ene & 22.5 & 20.0 & 4.8 & 50.2 & 21.9 & 241.5 & 119.1 & 47.6 & 66.0 \\
Feb & 70.2 & 30.8 & 26.8 & 80.2 & 63.2 & 22.1 & 0.0 & 49.8 & 42.9 \\
Mar & 58.4 & 22.4 & 17.4 & 5.4 & 2.8 & 66.9 & 0.0 & 20.6 & 24.2 \\
Abr & 11.2 & 49.7 & 67.4 & 17.0 & 34.4 & 39.1 & 18.5 & 43.4 & 35.1 \\
May & 2.5 & 47.4 & 56.2 & 133.2 & 3.0 & 80.9 & 91.1 & 104.2 & 64.8 \\
Jun & 8.3 & 23.6 & 18.6 & 4.5 & 26.0 & 0.0 & 34.2 & 23.2 & 17.3 \\
Jul & 1.5 & 1.2 & 0.0 & 3.5 & $\mathbf{9 . 6}$ & 0.0 & 30.4 & 0.0 & 5.8 \\
Ago & 0.0 & 53.4 & 0.0 & 1.7 & 3.2 & 0.0 & 66.7 & 0.0 & 15.6 \\
\hline Año & $\mathbf{4 0 7 . 9}$ & $\mathbf{3 8 6 . 3}$ & $\mathbf{3 7 2 . 8}$ & $\mathbf{5 1 6 . 8}$ & $\mathbf{3 3 0 . 6}$ & $\mathbf{7 2 0 . 1}$ & $\mathbf{7 2 3 . 4}$ & $\mathbf{9 2 2 . 8}$ & $\mathbf{5 4 7 . 6}$ \\
\hline Caract. & seco & seco & seco & normal & muy seco & muy & muy & muy & \\
año & & & & & & húmedo & húmedo & húmedo & \\
\hline
\end{tabular}

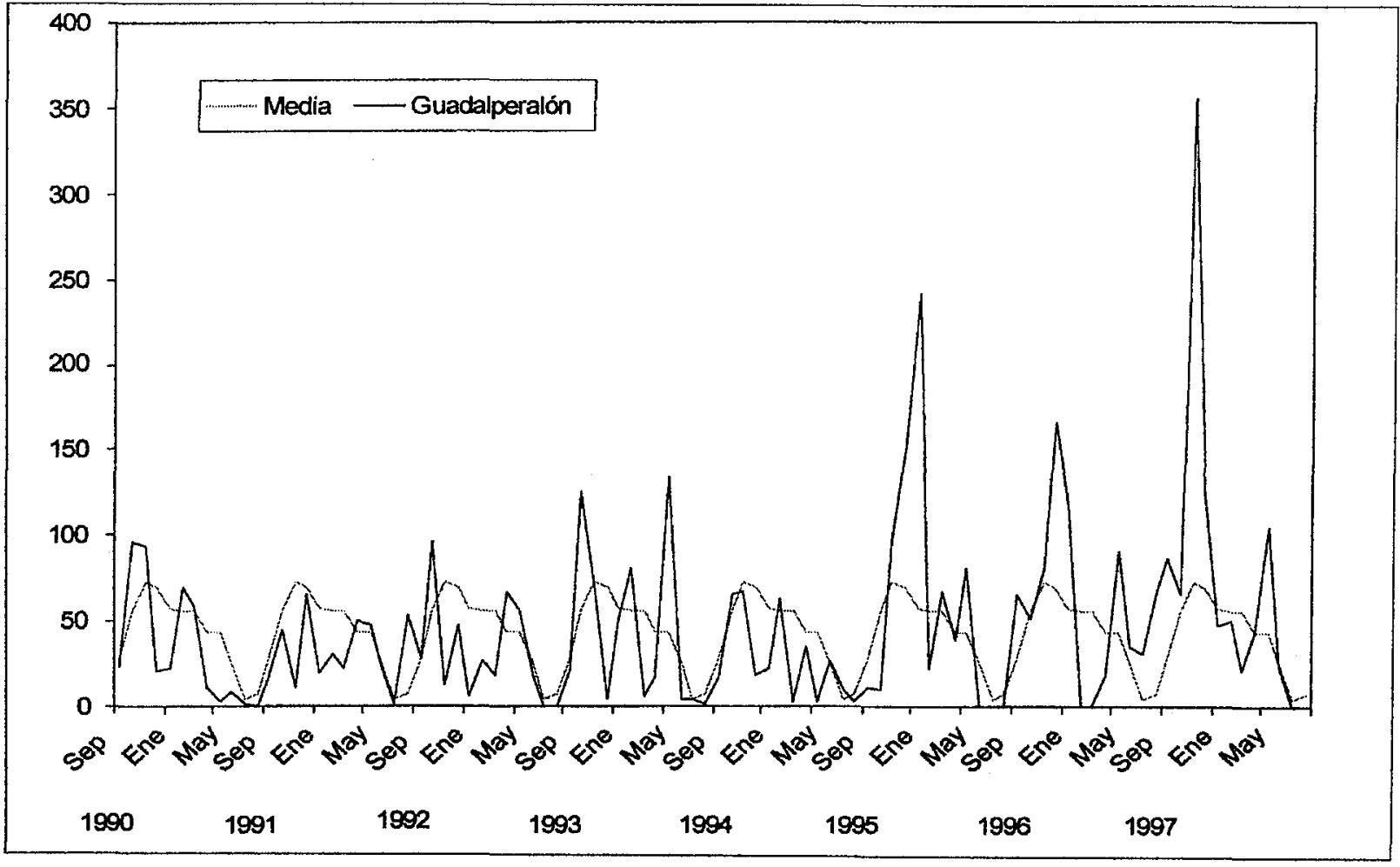

Figura 2. Precipitación mensual en Guadalperalón durante el período de estudio y medias aritméticas correspondientes a la serie del Observatorio de Cáceres (1907-1998, n=92). 


\subsection{Unidades edafo-vegetales}

Según la distribución del porcentaje de suelo desnudo, afloramientos rocosos, cobertura de pasto, cobertura de arbustos (Lavandula stoechas) y hojarasca se ha establecido una división espacial de la cuenca en 5 unidades de vegetación. La figura 3 representa las medias de cobertura para cada unidad, correspondiente a marzo de 1992. El análisis espacial de la densidad-porosidad y del contenido de agua del suelo a capacidad de campo coincidió con las unidades delimitadas, que son las siguientes (Schnabel, 1997; Ceballos \& Schnabel, 1998):

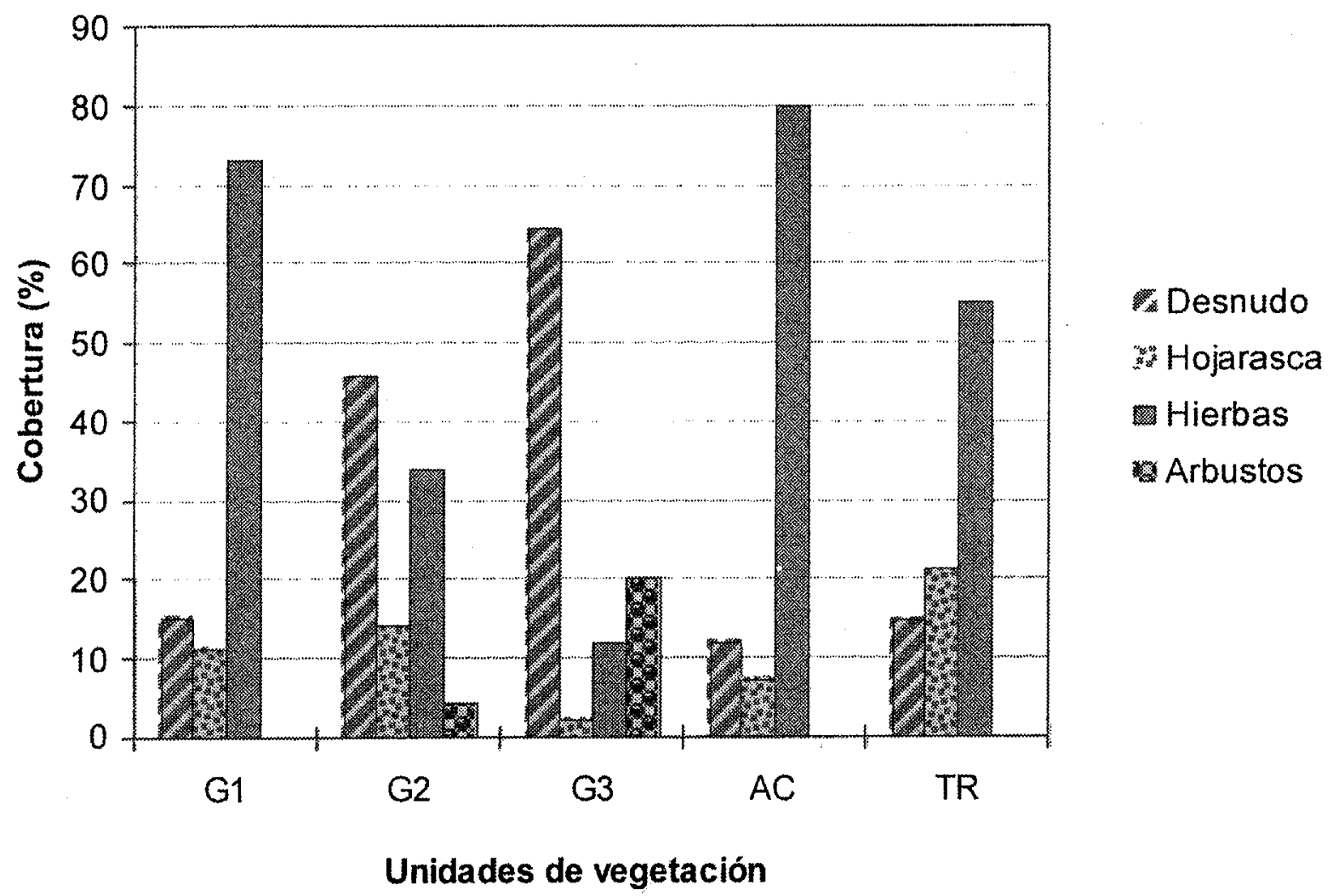

Figura 3. Cobertura media del suelo de las unidades de vegetación durante marzo de 1992 (Desnudo: suelo desnudo y afloramientos rocosos; Arbustos: principalmente Lavandula stoechas; Hierbas: plantas herbáceas).

G1. Representa el $7.8 \%$ de la superficie. La densidad de arbolado oscila entre los 30 y los 45 pies por hectárea. La cobertura de pastos es alta debido a la práctica del redileo (concentración del ganado en un aprisco durante la noche para que con sus excrementos fertilicen el terreno), por ello la porosidad media del suelo (51.9\%) y la capacidad de campo (29.7\%) son altas.

G2. Es la unidad con mayor superficie (50.9\%). La densidad del arbolado es muy variable (entre 5 y 45 pies por hectárea) y la cobertura de pastos menor que en la unidad anterior. Aparecen afloramientos de roca madre y manchas de Lavandula stoechas en las convexidades topográficas. Los suelos presentan una porosidad de $47.8 \%$ y una capacidad de campo de $26.2 \%$.

G3. Supone un $29.8 \%$ de la superficie y es el área más degradada de la cuenca. Presenta suelos poco profundos, afloramientos del sustrato pizarroso frecuentes (25\%), 
y el mayor porcentaje de superficie desnuda. El arbolado es inexistente. El pastizal es muy pobre y la presencia de $L$. stoechas es importante. Aparece también una costra liquénica superficial de espesor milimétrico en algunos puntos. Por ello la porosidad (47.5\%), así como la capacidad de campo (21.7\%) son las más bajas del conjunto.

AC. Comprende las zonas de pie de vertiente y las vaguadas rellenas de sedimentos fluvio-coluviales. La cubierta herbácea es densa con valores del 90\% durante años con pluviosidad media-alta. Su porosidad media es $48.2 \%$, aunque en la parte central del fondo de valle la porosidad alcanza el 58.3\% y los suelos, durante lluvias prolongadas, permanecen saturados varios días. Constituye el $11.5 \%$ de la superficie y carece de arbolado.

TR. Esta unidad superficialmente está incluida dentro de G1 y G2, ya que representa aquellas parcelas situadas bajo la proyección de la copa de las encinas. Tiene escasa superficie de suelo desnudo debido, no sólo al crecimiento de pasto, sino también a la existencia de una capa de hojarasca. Como consecuencia de la abundancia de materia orgánica, la porosidad y la capacidad de campo son muy altas, con unos valores medios respectivos de $63.0 \%$ y $39.2 \%$.

\subsection{Escorrentía superficial a escala de vertiente y de pedón}

Los datos de escorrentía superficial en las vertientes demuestran la existencia de dos grupos claramente diferenciados. Destacan los altos valores de las unidades G2 y G3 frente a los bajos valores de las unidades G1 y TR (Tabla 2). La cubierta vegetal, las características físicas del suelo y la posición topográfica se muestran como los principales factores que explican la distinta respuesta hidrológica de las unidades delimitadas. Las altas escorrentías producidas en G2 y G3 se relacionan con la degradación y escasa capacidad de infiltración de sus suelos. La unidad AC, salvo durante años muy húmedos, muestra unos valores más bajos respecto a G2 y G3. La posición topográfica de las parcelas pertenecientes a esta unidad (concavidades de las laderas y fondos de valle rellenos de sedimentos) explica que sea una de las zonas con mayores tasas de infiltración debido a la profundidad de los suelos, alto porcentaje y persistencia de pastos y menor densidad de sus suelos. No obstante, durante años muy húmedos (1995 y 1996), las precipitaciones continuadas provocan la saturación de estas zonas, con la consiguiente producción de flujo subsuperficial y de retorno, y con los registros más altos de escorrentía.

Contrastando con las unidades anteriores, G1 y TR registran los valores más bajos. En el caso de G1, la mayor porosidad de sus suelos y la mayor cobertura de pastos se relaciona con la práctica de redileo. En TR el factor determinante es la interceptación de la lluvia por el arbolado. Este hecho ha sido comprobado por los experimentos de infiltración con lluvia simulada, indicando bajas tasas de infiltración bajo las copas de las encinas. La realización de una serie de tests (WDTP) para estudiar el comportamiento hidrofóbico de los suelo ha demostrado una mayor repelencia bajo el influjo de las copas de los árboles que en las situadas fuera de su área de influencia (Cerdà et al., 1998). El comportamiento hidrofóbico se observó en mayo de 1995 cuando los suelos estaban secos, mientras que en febrero de 1996 el carácter repelente no se ha podido constatar debido a las lluvias prolongadas. 
Las dos series de experimentos de infiltración en microparcelas, realizadas en distintas condiciones de humedad antecedente y vegetación, ilustran acerca de la respuesta hidrológica de la cuenca ante diferentes situaciones (cambios estacionales) a escala de pedón. La diferenciación espacial de los procesos hidrológicos es mayor en ambiente seco que en ambiente húmedo, siendo la respuesta mucho más homogénea con la saturación de los suelos. La humedad antecedente es el factor más importante para explicar los cambios estacionales de los procesos hidrológicos a escala de pedón, hasta el punto de que de él dependen los modelos de flujo (flujo hortoniano en ambiente seco y flujo por saturación en ambiente húmedo) (Horton, 1940; Dunne \& Leopold, 1978); mientras que los cambios espaciales dependen de la cubierta vegetal. El uso del suelo es un factor que incide tanto en la variación estacional como espacial, apreciándose una diferencia del $36 \%$ en la infiltrabilidad de zonas pastoreadas frente a zonas no pastoreadas. A pesar de las divergencias estacionales y espaciales, los coeficientes de escorrentía son muy altos en comparación con otras zonas peninsulares, lo que se traduce en una escasa capacidad de infiltración de los suelos de la cuenca (Cerdà et al., 1998, Ceballos, 1999).

Tabla 2: Escorrentía superficial anual $\left(\mathrm{m}^{-1}\right)$ en vertientes y coeficientes de variación en las diferentes unidades espaciales, así como el número de veces en que ban rebosado los colectores de las parcelas (expresado como por ciento del número total de eventos). Los datos corresponden al periodo de septiembre 1990 basta noviembre 1993 con un total de 70 eventos de precipitación superior a $5.4 \mathrm{~mm}$ y 27 parcelas de erosión.

\begin{tabular}{lcccc}
\hline Unidad & $\mathrm{N}$ & $\begin{array}{c}\text { Escorrentía } \\
(1 / \text { m.año })\end{array}$ & $\begin{array}{c}\text { Coeficiente de variación } \\
(\%)\end{array}$ & $\begin{array}{c}\text { Colectores rebosados } \\
\text { (\% núm. eventos) }\end{array}$ \\
\hline G1 & 5 & 165.4 & 24.0 & 3.7 \\
G2 & 7 & 422.8 & 37.3 & 15.7 \\
G3 & 6 & 501.8 & 56.9 & 10.5 \\
AC & 5 & 188.7 & 23.5 & 6.3 \\
TR & 4 & 133.5 & 29.9 & 4.3 \\
Total & 27 & 306.5 & 71.4 & 8.4 \\
\hline
\end{tabular}

\subsection{Interceptación}

Las encinas interceptan como promedio un $26.7 \%$ de la precipitación anual. Los árboles con un mayor desarrollo de sus copas (no podados) registran valores más elevados de interceptación (29.9\%) que los podados (23.6\%). La cantidad de escorrentía cortical es muy baja y representa menos del 1\% de la aportación anual de lluvia. Para determinar el papel del arbolado en el balance de agua para toda la cuenca hay que considerar el grado de superficie cubierta por el arbolado, que ha sido estimado en un $8 \%$. Por tanto, la pérdida de agua por interceptación de las encinas en Guadalperalón supone un $4 \%$ de la precipitación anual.

\subsection{Caudales generados a nivel de cuenca}

Precipitaciones superiores a $9 \mathrm{~mm}$ y con intensidades máximas en 10 minutos de aproximadamente $25 \mathrm{~mm} \mathrm{~h}^{-1}$ han generado caudal. Sucesos de precipitación de 
moderada-alta intensidad y cantidades hasta $25 \mathrm{~mm}$ producen flujo de corta duración (3 horas o menos) con tiempos de pico (tiempo entre máximo de lluvia y máximo de caudal) de entre 5 y 15 minutos (Fig. 4). El hidrograma típico observado en Guadalperalón ofrece una curva de crecida con pendiente muy pronunciada y un descenso algo menos rápido (Fig. 4). Este hecho explica que en la mayoría de los casos más del $50 \%$ del volumen total de caudal se produzca en un período de tiempo inferior a una hora. Estos hidrogramas reflejan la generación de flujo de tipo Hortoniano, donde la intensidades de precipitación superan la capacidad de infiltración de los suelos en las vertientes.

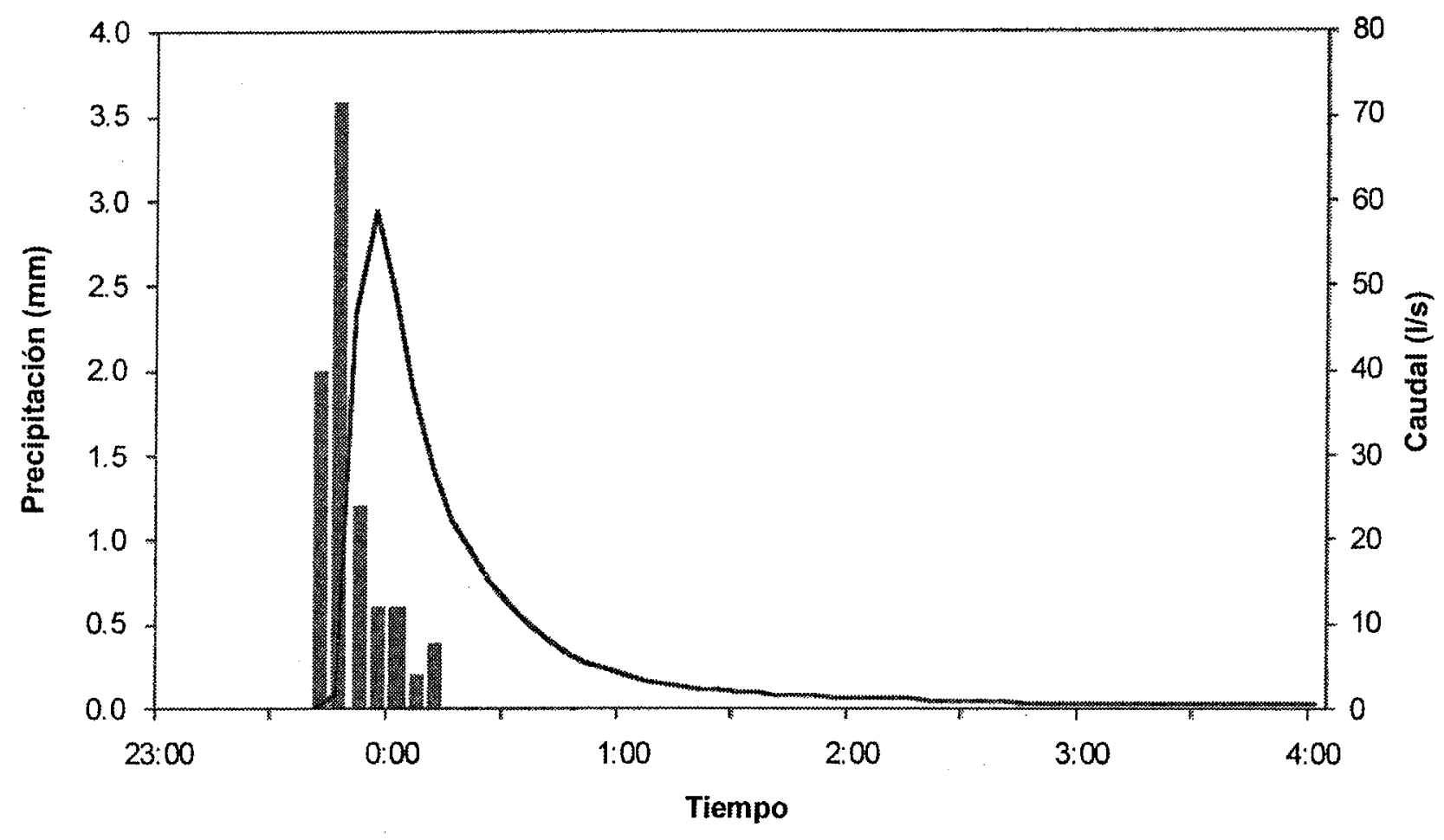

Figura 4. Típico bidrograma de una crecida registrada en la salida de la cuenca (resolución temporal: 5 minutos).

En tres ocasiones el flujo en el cauce sobrepasó la capacidad del aforador (860 $1 \mathrm{~s}^{-1}$ ), debido a sucesos de precipitación de gran intensidad y cantidad (Tabla 3). En dos de ellos se ha podido estimar el caudal máximo aplicando la ecuación de Manning y perfiles transversales, correspondientes a los nivelos máximos de agua indicados por depósitos en las orillas del cauce (Ceballos \& Schnabel, 1999). Los picos de caudal estimados en aproximadamente $4 \mathrm{~m}^{3}$ corresponden a caudales específicos de $11 \mathrm{~m}^{3} \mathrm{~s}^{-1} \mathrm{~km}^{-2}$, valores que pueden ser considerados altos en comparación con las crecidas extremas citadas en García Ruiz et al. (1996). 
Tabla 3: Eventos de caudal registrados en la salida de la cuenca con aportaciones superiores a $50 \mathrm{~m}^{3}$. Q-max: máximo caudal en 5 minutos, R.C.: coeficiente de escorrentía, I30: máxima intensidad en 30 minutos (* - flujo continuo, $\$$ - estimación, - falta de datos).

\begin{tabular}{|c|c|c|c|c|c|c|}
\hline \multicolumn{2}{|c|}{ Date } & Aportación & $Q-\max$ & R.C. & Precipitación & $1-30$ \\
\hline Año $91-92$ & $\begin{array}{l}01 / 12 / 91 \\
30 / 03 / 92 \\
07 / 08 / 92 \\
\end{array}$ & $\begin{array}{r}228.6 \\
88.6 \\
\$ 883.5 \\
\end{array}$ & $\begin{array}{r}41.9 \\
49.5 \\
>860.0 \\
\end{array}$ & $\begin{array}{r}2.2 \\
1.6 \\
11.7 \\
\end{array}$ & $\begin{array}{l}30.2 \\
15.6 \\
21.6 \\
\end{array}$ & $\begin{array}{r}7.6 \\
12.4 \\
32.8 \\
\end{array}$ \\
\hline Año $92-93$ & $\begin{array}{l}26 / 09 / 92 \\
11 / 10 / 92 \\
16 / 10 / 92 \\
19 / 10 / 92 \\
29 / 10 / 92 \\
14 / 04 / 93 \\
24 / 04 / 93 \\
03 / 05 / 93 \\
\end{array}$ & $\begin{array}{r}215.9 \\
149.4 \\
79.7 \\
198.7 \\
347.2 \\
145.2 \\
280.7 \\
59.3 \\
\end{array}$ & $\begin{array}{r}86.9 \\
62.2 \\
69.3 \\
73.5 \\
226.3 \\
74.2 \\
295.0 \\
24.1 \\
\end{array}$ & $\begin{array}{l}2.3 \\
4.6 \\
1.9 \\
3.0 \\
6.3 \\
5.3 \\
3.9 \\
1.8 \\
\end{array}$ & $\begin{array}{r}26.4 \\
9.2 \\
12.2 \\
19.0 \\
15.8 \\
7.8 \\
20.4 \\
9.2 \\
\end{array}$ & $\begin{array}{r}12.8 \\
5.4 \\
9.2 \\
8.0 \\
14.4 \\
14.4 \\
14.2 \\
16.4 \\
\end{array}$ \\
\hline $\begin{array}{l}\text { Año in- } \\
\text { completo }\end{array}$ & $\begin{array}{l}15 / 05 / 94 \\
21 / 05 / 94 \\
\end{array}$ & $\begin{array}{l}163.6 \\
948.0 \\
\end{array}$ & $\begin{array}{l}139.6 \\
125.0 \\
\end{array}$ & $\begin{array}{l}2.0 \\
5.3\end{array}$ & \begin{tabular}{|l|}
23.6 \\
50.8 \\
\end{tabular} & $\begin{array}{l}11.2 \\
22.8 \\
\end{array}$ \\
\hline Año 94.95 & $\begin{array}{l}05 / 10 / 94 \\
28 / 10 / 94 \\
12 / 02 / 95 \\
28 / 04 / 95 \\
\end{array}$ & $\begin{array}{r}57.5 \\
59.9 \\
1887.0 \\
112.4 \\
\end{array}$ & $\begin{array}{r}122.3 \\
20.3 \\
81.7 \\
92.7 \\
\end{array}$ & $\begin{array}{r}0.8 \\
1.3 \\
13.1 \\
2.1 \\
\end{array}$ & $\begin{array}{l}27.4 \\
16.2 \\
40.8 \\
19.0 \\
\end{array}$ & $\begin{array}{r}22.6 \\
9.8 \\
7.4 \\
17.1 \\
\end{array}$ \\
\hline Año $95-96$ & $\begin{array}{c}25 / 12 / 95 \\
* 27 / 12 / 95 \\
-17 / 02 / 96 \\
15 / 03 / 96 \\
28 / 03 / 96 \\
08 / 05 / 96 \\
11 / 05 / 96 \\
\end{array}$ & $\begin{array}{r}432.6 \\
34100.0 \\
\\
270.8 \\
383.5 \\
58.4 \\
809.5 \\
\end{array}$ & $\begin{array}{r}126.0 \\
\$ 4050.0 \\
\\
26.6 \\
46.7 \\
22.0 \\
277.0 \\
\end{array}$ & $\begin{array}{r}7.7 \\
27.9 \\
\\
2.7 \\
4.9 \\
1.4 \\
8.0 \\
\end{array}$ & \begin{tabular}{r|}
20.4 \\
344.5 \\
\\
28.4 \\
22.3 \\
11.2 \\
28.5 \\
\end{tabular} & \begin{tabular}{r|}
12.4 \\
33.6 \\
\\
5.6 \\
16.4 \\
13.6 \\
28.8 \\
\end{tabular} \\
\hline Año 96-97 & $\begin{array}{c}13 / 10 / 96 \\
11 / 11 / 96 \\
13 / 11 / 96 \\
* 12 / 12 / 96 \\
-31 / 01 / 97 \\
24 / 08 / 97\end{array}$ & $\begin{array}{r}112.5 \\
100.8 \\
180.1 \\
43188.8\end{array}$ & $\begin{array}{r}40.3 \\
23.3 \\
111.6 \\
163.9 \\
\$ 4250.0\end{array}$ & $\begin{array}{r}0.8 \\
0.7 \\
2.6 \\
47.5\end{array}$ & $\begin{array}{r}39.8 \\
42.2 \\
19.8 \\
257.4\end{array}$ & $\begin{array}{l}12.4 \\
14.0 \\
22.4 \\
16.4\end{array}$ \\
\hline
\end{tabular}

Las características principales de los eventos de caudal registrados en Guadalperalón se presentan en la tabla 3, incluyendo solamente aquellos con aportaciones superiores a $50 \mathrm{~m}^{3}$, ya que valores inferiores tienen muy poca importancia en el balance anual de agua. Durante los tres años secos (1991, 1992 y 1994) se han observado solamente 5 casos por año, como promedio, en los cuales hubo escorrentía en el cauce principal. Durante los inviernos de 1995 y 1996 se registraron un gran número de días de lluvia que aportaron cantidades de agua elevadas. Como consecuencia se produjo una saturación de los suelos en las vertientes y también de los sedimentos en los fondos de valle, causando la generación de caudales abundantes y flujo continuo en el cauce principal (Tabla 3).

Los fondos de valle, por su capacidad de retener agua, son el elemento determinante para explicar los caudales generados en la salida de la cuenca, las características de los hidrogramas y el modelo de escorrentía. Según la continuidad y la cantidad de las precipitaciones variará temporalmente el porcentaje de humedad de estas zonas, que pueden identificarse como áreas fuente de escorrentía de la 
cuenca. En estado seco y con alta capacidad de retención hídrica el modelo será el hortoniano. Los coeficientes de escorrentía son bajos y los hidrogramas recogen la escasa duración y alta pendiente de los tramos de crecida y recesión. En situación de precipitaciones abundantes y continuadas (años muy húmedos), y cuenca saturada, el tipo de escorrentía dominante será el de flujo de saturación y de retorno. Los coeficientes serán mayores y los hidrogramas se caracterizarán por una larga duración debido, sobre todo, a la prolongación de la curva de recesión.

La tabla 4 representa los valores mensuales de precipitación, aportación y coeficiente de escorrentía para el año hidrológico 1996, que ilustra la importancia de las condiciones antecedentes de humedad sobre las aportaciones. A los meses de diciembre y enero correspondió el $96 \%$ del total anual de caudal, debido no solamente a las lluvias producidas en dichos meses, sino también a la gran cantidad de precipitación caída durante los meses anteriores $(225 \mathrm{~mm}$ ) (Fig. 5).

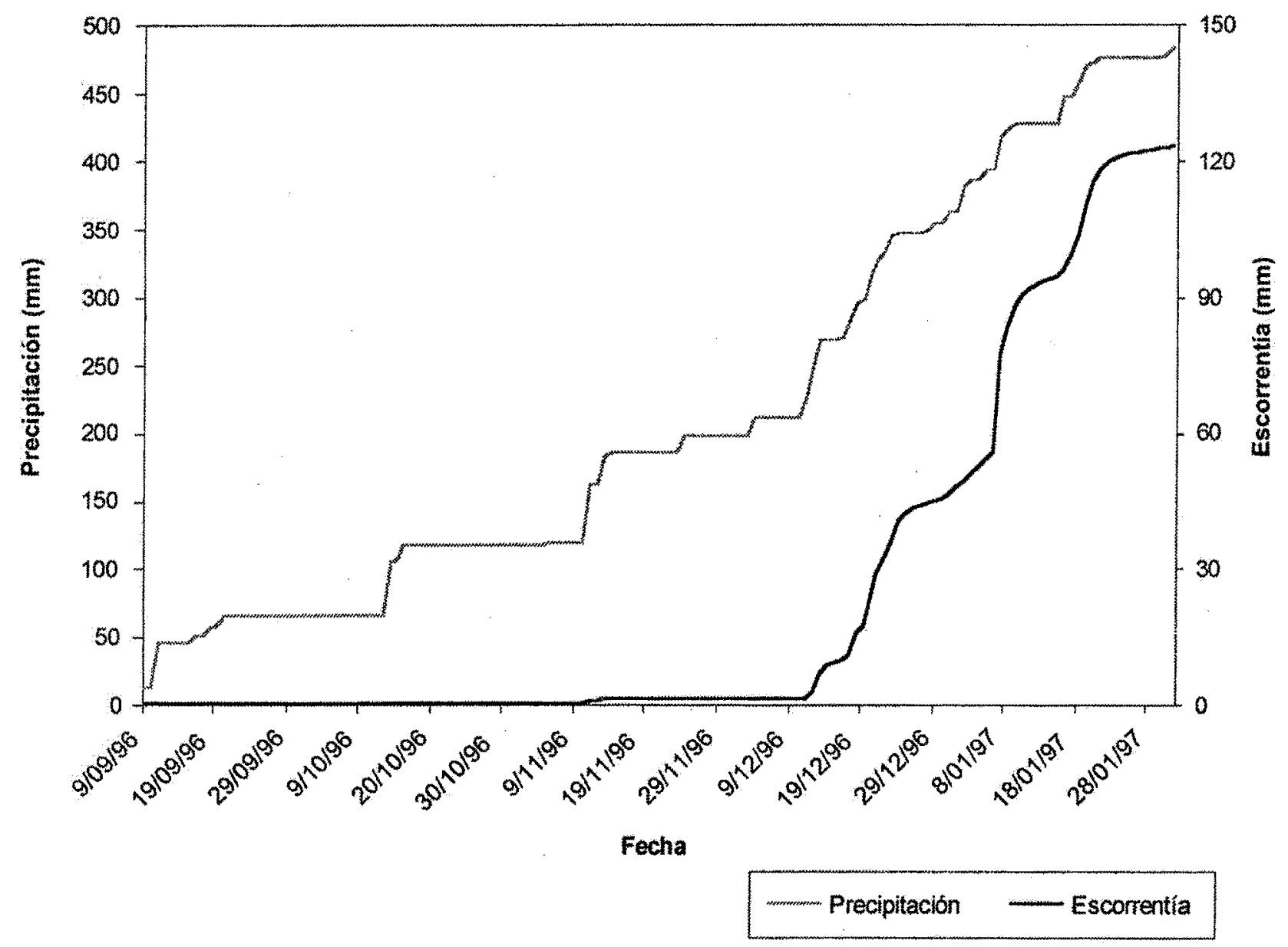

Figura 5. Cantidades de precipitación y aportaciones acumuladas para el período comprendido entre septiembre de 1997 y enero de 1998. 
Tabla 4: Valores mensuales de precipitación, escorrentía (aportaciones), caudal máximo en 5 minutos y coeficiente de escorrentía durante el año 1996-97

(*-valor estimado)

\begin{tabular}{lrrrr}
\hline $\begin{array}{l}\text { 1996-97 } \\
\text { Mes }\end{array}$ & $\begin{array}{c}\text { Precipitación } \\
(\mathrm{mm})\end{array}$ & $\begin{array}{c}\text { Escorrentia } \\
(\mathrm{mm})\end{array}$ & $\begin{array}{c}\text { Caudal máximo } \\
(\mathrm{l} / \mathrm{s})\end{array}$ & $\begin{array}{c}\text { Coeficiente de esco. } \\
(\%)\end{array}$ \\
\hline Sep & 65.1 & 0.0 & 0.0 & 0.0 \\
Oct & 51.8 & 0.3 & 40.3 & 0.6 \\
Nov & 80.2 & 0.8 & 116.6 & 1.0 \\
Dic & 166.3 & 45.5 & 102.1 & 27.3 \\
Ene & 119.1 & 76.5 & 163.9 & 64.3 \\
Feb & 0.0 & 0.0 & 0.0 & 0.0 \\
Mar & 0.0 & 0.0 & 0.0 & 0.0 \\
Abr & 18.5 & 0.0 & 0.0 & 0.0 \\
May & 91.1 & 0.2 & 31.5 & 0.3 \\
Jun & 34.2 & 0.2 & 12.7 & 0.5 \\
Jul & 30.4 & 0.0 & 0.0 & 0.0 \\
Ago & 66.7 & $* 3.6$ & 4250.0 & 5.4 \\
\hline Año & 723.4 & 127.1 & 4250.0 & 17.6 \\
\hline
\end{tabular}

\subsection{Balance de agua}

Los coeficientes de escorrentía de la cuenca han sido muy variables, con valores muy bajos durante los años secos (1.5\%) y valores más altos durante años húmedos (15.9\%). La media se sitúa en un 7.3\%. Estos valores son comparables a los obtenidos en otras zonas con condicones climáticas parecidas (por ejemplo Domingo, et al., 1994; Piñol et al., 1991). Los balances de agua anuales se presentan en la tabla 5. Las pérdidas por evapotranspiración media han sido de aproximadamente $90.3 \%$, similar a valores citados para otras cuencas situadas en áreas de clima semiárido (Dubreuil, 1985; Rodier, 1975).

El análisis comparativo de los procesos hidrológicos para las diferentes escalas analizadas indica una correlación negativa entre el coeficiente de escorrentía y el tamaño de la superficie de medición debido a los mecanismos de reinfiltración (Ceballos et al., 1998). Conforme aumenta el tamaño de la superficie productora de escorrentía, más compleja resulta la distinción de los factores que determinan el flujo superficial. La comparación de los resultados de las diferentes escalas demuestra la importancia clave de los fondos de valle rellenos con sedimentos coluvio-fluviales sobre el comportamiento hidrológico global de la cuenca. No obstante, los datos disponibles para la cuenca Guadalperalón son todavía insuficientes para entender y cuantificar las relaciones entre diferentes escalas espaciales, debido a algunas limitaciones técnicas de medición (desbordamiento de los colectores, problemas del aforador). 
Tabla 5: Balances de agua anuales y coeficientes de escorrentía.

\begin{tabular}{ccccc}
\hline Año & $\begin{array}{c}\text { Precipitación } \\
(\mathrm{mm})\end{array}$ & $\begin{array}{c}\text { Escorrentía } \\
(\mathrm{mm})\end{array}$ & $\begin{array}{c}\text { Evapotransp.real } \\
(\mathrm{mm})\end{array}$ & $\begin{array}{c}\text { Coef. Esco. } \\
(\%)\end{array}$ \\
\hline $1991-92$ & 386.3 & 3.9 & 382.1 & 1.0 \\
$1992-93$ & 372.8 & 4.9 & 367.9 & 1.3 \\
$1994-95$ & 330.6 & 7.5 & 323.1 & 2.3 \\
$1995-96$ & 720.1 & 102.3 & 617.8 & 14.2 \\
$1996-97$ & 723.4 & 127.1 & 596.3 & 17.6 \\
Media & 506.6 & 49.1 & 457.4 & 7.3 \\
\hline
\end{tabular}

\section{Agradecimientos.}

Este trabajo ha sido financiado por los proyectos AMB 92-0580, AMB95-0986-C02-02 y HID98-1056-C02-02 (CICYT) y RESEL (Dirección General de Conservación de la Naturaleza). Los autores agradecen a los Dres. F. Gallart y P. Llorens el apoyo prestado durante la investigación.

\section{Bibliografia}

BERNET HERGUIJUELA, R., 1995. La cubierta berbácea en sistemas de debesa degradados. Universidad de Extremadura y FUNDICOT, Cáceres, .

CEBALlOS, A., 1999. Procesos bidrológicos en una pequeña cuenca bidrográfica bajo explotación de debesa en Extremadura. Universidad de Extremadura, Cáceres, 196 pp.

CEBALLOS, A. \& SCHNABEL, S., 1998. Hydrological behaviour of a small catchment in the dehesa landuse system (Exremadura, SW Spain). Journal of Hydrology, 210, 146-160.

CEBAllos, A. \& SCHNABEL, S., 1998. Comportamiento de la humedad del suelo en una pequeña cuenca hidrográfica de la dehesa extremeña (Guadalperalón, Cáceres). Cuadernos de Investigación Geográfica, 24, 25-38.

CEBALlOS, A., SCHNABEL, S., GÓMEZ AMELIA, D. \& CERDÀ, A., 1998. Relación entre la escala espacial y escorrentía superficial en una pequeña cuenca hidrográfica semiárida ante condiciones contrastadas de humedad del suelo (Extremadura, sO de España). Cuaternario y Geomorfología, 12, 63-75.

CERDÀ, A., SCHNABEL, S., CEBALLOS, A. \& GÓMEZ AMELIA, D., 1998. Soil hydrological response under simulated rainfall in the dehesa land system (Extremadura, SW Spain) under drought conditions. Earth Surface Processes and Landforms 23, 195-209.

DIÁZ, M., CAMPOS, P. \& PULIDO, F.J., 1997. The Spanish dehesa: a diversity in land-use and wildlife. En: Farming and Birds in Europe (D.J. Pain \& M.W. Pienkowski, eds.). Academic Press, San Diego, 178-209.

DOMINGO, F., PUIGDEFÁBREGAS, J., MORO, M.J. \& BELLOT, J., 1994. Role of vegetation cover in the biogeochemical balances of a small afforested catchment in southeastern Spain. Journal of Hydrology, 159, 275-289.

DUBREUIL, P.L., 1985. Review of field observations of runoff generation in the tropics. Journal of Hydrology, 80: 237-264. 
DUNNE, T. \& LEOPOLD, L. B., 1978. Water in Environmental Planning. Freeman \& Company, New York, 818 pp.

ELENA, M., LÓPEZ, J.A., CASAS, M. \& SÁNCHEZ, A., 1987. El carbón y la encina. Instituto Nacional de Investigaciones Agrarias, MAPA, Madrid.

FAO (Food and Agriculture Organization) (1990): Soil Map of the World 1:5.000.000, revised legend. FAO, Rom.

FONT TULLOT, I., 1983. Climatología de España y Portugal. Instituto de Meteorología, Madrid.

GAMOUGOUN, N.D., SMITH, R.P., WOOD, K. \& PIEPER, R.D., 1984. Soil vegetation and hydrologic responses to grazing management at Fort Stanton, New Mexico. Journal of Range Management, 37: 538-541.

GARCÍA RUIZ, J.M., WHITE, S.M., MARTÍ, C., VALERO, B., ERREA, M.P. \& GÓMEZ VILLAR, A., 1996. La catástrofe del Barranco de Arás (Biescas, Pirineo Aragonés) y su contexto espacio-temporal. Instituto Pirenáico de Ecología, CSIC, Zaragoza, 54 pp.

GÓMEZ AMELIA, D., 1985. La penillanura cacereña. Estudio geomorfológico. Universidad de Extremadura, Cáceres.

GÓMEZ AMELIA, D. \& SCHNABEL, S., 1992. Procesos sedimentológicos e hidrológicos en una pequeña cuenca bajo explotación de dehesa en Extremadura. En: Estudios de Geomorfología en España (F. López Bermúdez, C Conesa García \& M.A. Romero Díaz, eds.), Sociedad Española de Geomorfología, Murcia, 55-63.

GÓMEZ AMELIA, D. \& SCHNABEL, S. 1996. Hidrología y erosión en ambientes de pastoreo extensivo. En: Erosión y recuperación de tierras en áreas marginales (T. Lasanta, \& J.M. García Ruiz, eds.), Instituto de Estudios Riojanos y Sociedad Española de Geomorfología, Logroño, 137-154.

HOLECHEK, J.L., PIEPER, R.D. \& HERBEL, C.H., 1989. Range Management, Principles and Practices. Prentice-Hall, Englewood Cliffs, New Jersey.

HORTON, R.E., 1940. An approach toward a physical interpretation of infiltration capacity. Proc. Soil Sci. Soc, Am., 5: 399-417.

I.G.M.E., 1987. Mapa Geológico de España. Hoja núm. 679 (Aldea de Trujillo). Instituto Geológico Minero de España, Madrid.

IMESON, A.C., 1988. Una vía de ataque eco-geomorfológica al problema de la degradación y erosión del suelo. En: Desertificación en Europa (MOPU, ed.), Madrid, 161-181.

LADERO ÁLVAREZ, M., 1987. La España Luso-Extremadurense. En: La vegetación de España. (M. Peinado Lorca \& S. Rivas-Martínez, eds.). Colección Aula Abierta, Universidad de Alcalá de Henares, 455-488.

MATEOS RODRÍGUEZ, A.B. \& SCHNABEL, S. 1998. Medición de la interceptación de las precipitaciones por la encina (Quercus rotundifolia Lam.): Metodología e instrumentalización. Norba Geografía, 10, 95-112.

MONTOYA OLIVER, J.M., MESÓN GARCÍA, M.L. \& RUIZ DEL CASTILLO, J., 1988. Una debesa testigo. Instituto Nacional para la Conservación de la Naturaleza, Madrid, $134 \mathrm{pp}$.

MULHOLLAND, B. \& FULLEN, M.A., 1991. Cattle trampling and soil compaction on loamy sands. Soil Use and Management, 7, 189-193.

PIÑOL, J., LLEDÓ, M.J. \& ESCARRÉ, A., 1991. Hydrological balance of two mediterranean forested catchments (Prades, norheast Spain). Hydrological Sciences-Journal-des Sciences Hydrologiques, 36 (2-4), 97-107. 
RODIER, J., 1975. Evaluation of annual runoff in tropical African Sabel. ORSTOM Document, 145.

SCHNABEL, S., 1997. Soil erosion and runoff production in a small watersbed under silvopastoral landuse (dehesas) in Extremadura, Spain. Geoforma Ediciones, Logroño, 167 pp.

SCHNABEL, S., 1998. La precipitación como factor en los procesos hidrológicos y erosivos análisis de datos de Cáceres capital. Norba Geografía, 10, 137-153.

SCHNABEL, S., GÓMEZ AMELIA, D. \& CEBALlOS BARBANCHO, A., 1999. Extreme events and gully erosion. Proceedings of the International Seminar on Land Degradation and Desertification, International Geographical Union, Lisboa 1988, 17-26.

SHARON, D., 1970. Areal patterns of rainfall in a small watershed. Symposium Results of Research on Representative and Experimental Basins. IAHS Publ., 96, 3-11.

USDA, 1979. Field Manual for Research in Agricultural Hydrology. Agriculture Handbook $\mathrm{n}^{\circ} 224$, United States Department of Agriculture.

Recibido en noviembre del 2000 Aceptado en enero del 2001 